\title{
Mating Behavior and Oviposition in the Pycnogonid Propallene longiceps
}

\author{
K. Nakamura ${ }^{1}$ and K. Sekiguchi ${ }^{2}$ \\ ${ }^{1}$ Kunitachi Senior High School, Kunitachi-shi, Tokyo, Japan* \\ 2 The University of Tsukuba, Institute of Biological Sciences, Sakura-mura, Ibaraki, Japan
}

ABSTRACT: Mating behavior and oviposition in the pycnogonid Propallene longiceps (Böhm) were observed in the laboratory. The female collects the eggs laid and transfers them to the male. The male forms bracelet-like egg masses and carries them on its ovigerous legs until hatching. The processes of copulation and oviposition are described in detail and compared with those reported for other, related species.

\section{INTRODUCTION}

The pycnogonid Propallene longiceps (Böhm) is abundant in the vicinity of Nabeta Bay near Shimoda Marine Biological Station of The University of Tsukuba, Shizuoka, Japan (Sekiguchi et al., 1971). In pycnogonids, males carry the eggs laid until the larvae hatch. Very few papers report on the mating behavior of pycnogonids (Hoek, 1881; Cole, 1901; Sanchez, 1959). In P. longiceps, copulation and oviposition could be observed under laboratory conditions, when adult males and females were cultivated together in the same container. More than 30 cases of copulation with identical behavior patterns were recorded. Mating behavior, oviposition, and egg-mass formation in $P$. longiceps are described in detail.

\section{MATERLAL AND METHODS}

Adult male and female Propallene longiceps (Fig. 1) were collected by a specially designed net devised by Hajime Ueda of the Shimoda Marine Biological Station. The net was made of $2 \times 2 \mathrm{~mm}$ nylon mesh with a zinc-coated iron bar $(2.6 \mathrm{~m})$ horizontally attached to the mouth to open the net while towed over the sandy bottom. The net was towed as slowly as possible for about $500 \mathrm{~m}$ at water depths ranging from 7 to $15 \mathrm{~m}$. The pygnogonids were isolated from the sand by a sieve and classified according to species, sex and age.

- Mailing address: Dr. Koichiro Nakamura 2-25-3, Nakamachi, Musashino-shi, Tokyo, 180, Japan.
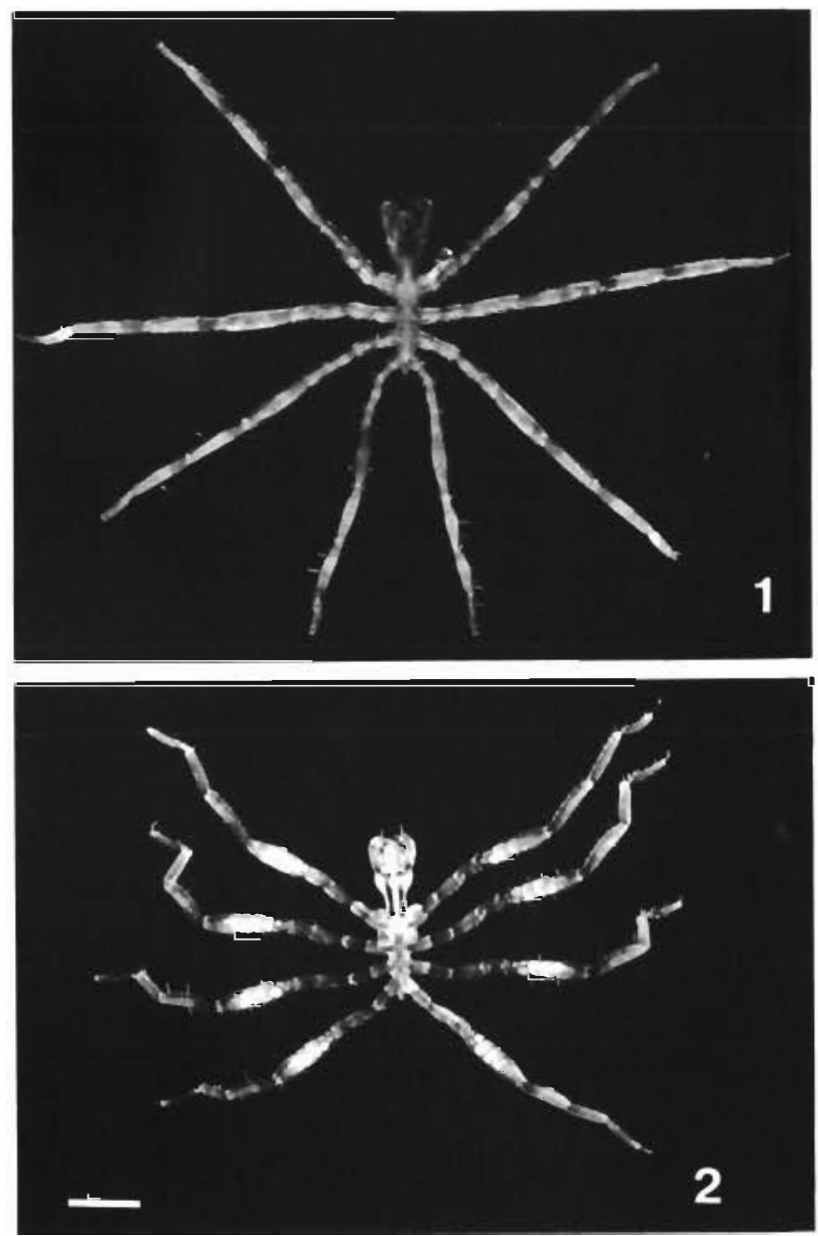

Fig. 1. Propallene longiceps. Adult male (1) and female (2). Scale bar $=1 \mathrm{~mm}$ 
Adults were cultivated in plastic or glass containers of $90 \mathrm{~mm}$ diameter with seawater of $26^{\circ}$ to $28^{\circ} \mathrm{C}$, and fed young Artemia salina.

In order to stain the eggs in its femur, the whole body of a female pycnogonid was stained with $0.0005 \%$ Nile blue or $0.001 \%$ neutral red for $30 \mathrm{~min}$. The eggs retained the color even after oviposition. Therefore, it was possible to determine which female had mated with the male by the color of the eggs carried.

\section{RESULTS}

From copulation to the transfer of the eggs laid, from the female to the male, five steps can be distinguished:
(1) The male holds the female thus stimulating oviposition; (2) from the ovary in the femur of the female mature eggs are released and forced toward the genital pore inside the ambulatory leg; (3) the female holds the eggs with her ovigerous legs; (4) the eggs are transferred from female to male; and (5) the male begins to form a bracelet-like egg mass around his ovigerous leg. The eggs are carried by the male until they hatch.

S te p 1: The male attaches to the dorsal side of the female, head in the same direction as the female, and holds her at the base of her chelifores with his own chelifores. Four distal segments of the male's ovigerous legs cling to the female's second ambulatory legs.

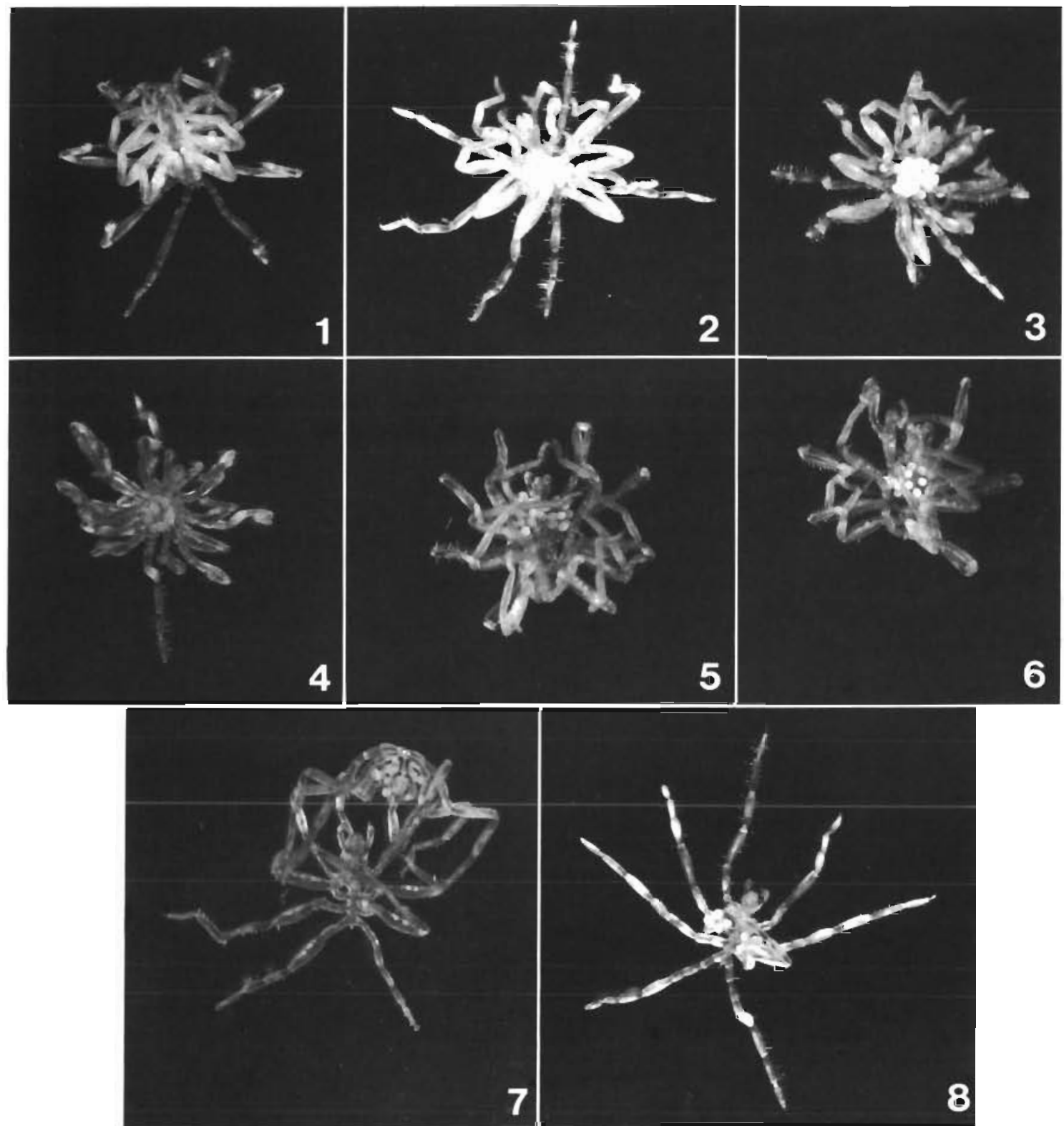

Fig. 2. Propallene longiceps. Copulation and oviposition. 1: male and female in mating position (Step 2); 2 : Step 3; 3 to 7 : Step $4 ;$ 8: Step 5 - the male holds the eggs. For details consult text 
Sometimes, the male holds the female at the propodus of her first and second ambulatory legs employing the claws of his corresponding legs. The male moves his ovigerous legs rhythmically, folding and stretching them, while the female folds all her ambulatory legs. In the laboratory, mating couples were usually found in this position (Fig. 2-1). Lying on his back, the male holds the female to his ventral side. This mating position was often maintained for more than $1 \mathrm{~h}$; it is not known how long this period lasts generally.

Sometimes, two males were observed assuming the mating position. On one occasion, an adult male and a subadult female joined in the mating position. Therefore, it seems that the male takes a leadership in the initiation of copulation.

St e p 2 (Figs 2-1, 3-1): The ovaries are located in the femur of each ambulatory leg of the female. There are usually two mature eggs in the center of each ovary (Fig. 4-1). Fully matured eggs occupy almost the entire width of the femur. During copulation, the mature eggs are released from the ovary one by one (Fig. 4). As the space outside the ovary is very narrow, the eggs become flattened; after a while they begin to move toward the base of the ambulatory leg inside the femur
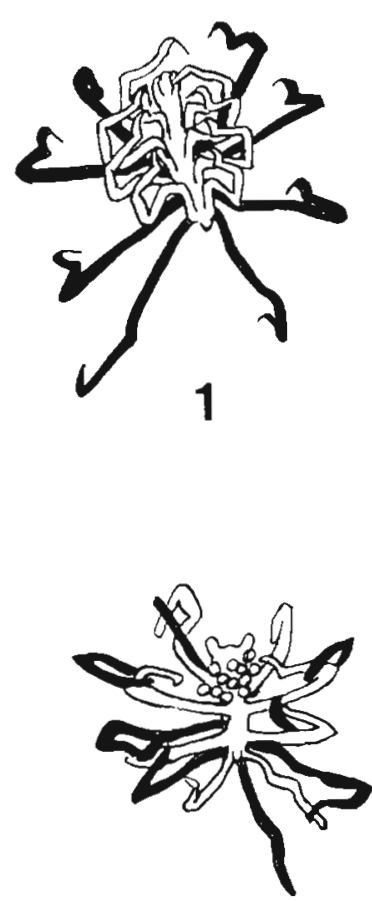

4

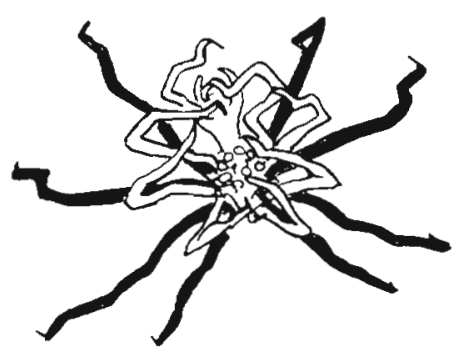

2

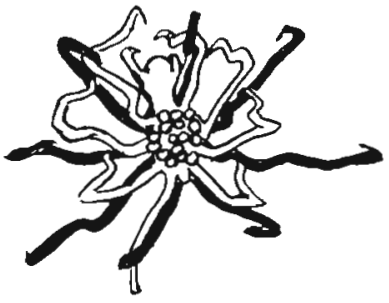

3

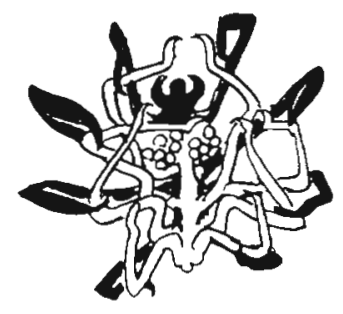

5

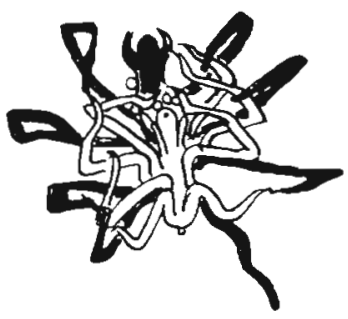

6
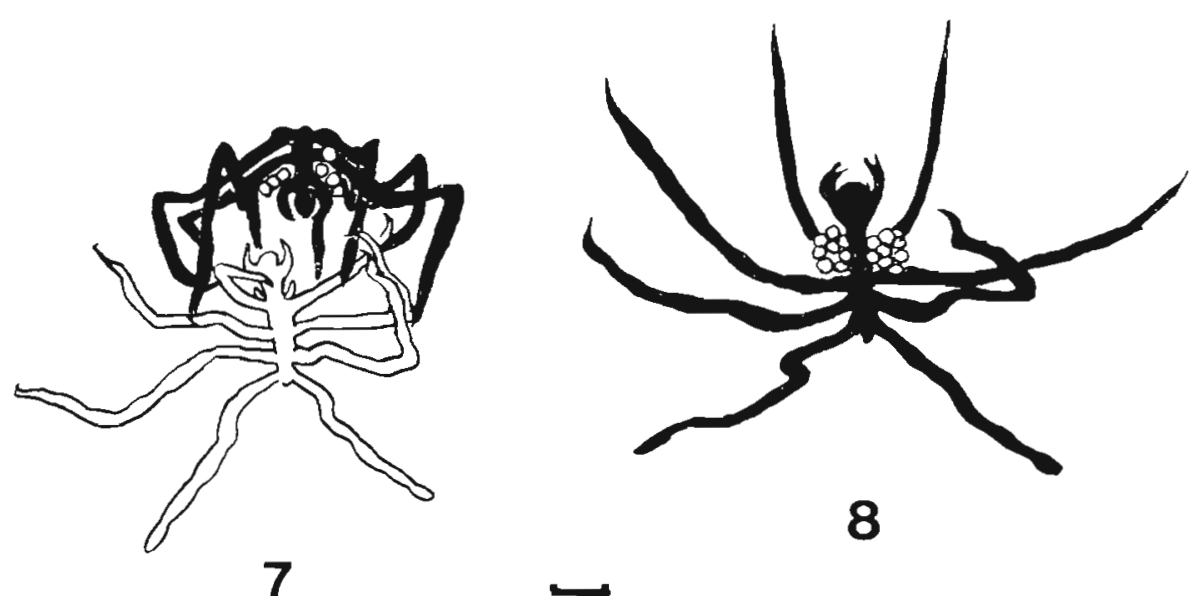

8

Fig. 3. Propallene longiceps. Copulation and oviposition. Schematic interpretation of photos in Figure 2 . Scale bar $=0.8 \mathrm{~mm}$ 
(Fig. 4-2). This egg transfer seems to be caused by peristaltic movements of the diverticula. The egg passes the third coxa and reaches the genital pore which is located in the second coxa (Fig. 4:3 and 4). Finally, the egg is forced out through the genital pore. The two eggs in each femur are released one by one with some interval. It takes about 10 to $25 \mathrm{~min}$ from the first egg's release to its delivery. The duration of this process varies, primarily because the eggs stay in the femur for different lengths of time. Once an egg begins to move, it comes out from the genital pore within about $3 \mathrm{~min}$. Then, the second egg follows about 3 to 5 min later.

Step 3 (Figs 2-2, 3-2): The female collects and holds the eggs laid with her ovigerous legs. At this time, the male bends the fourth ambulatory legs to bring the second coxa, where the genital pore opens, near the eggs laid. He keeps this position for about 5 to 10 min with his ovigerous legs moving actively. During this period we have, in several cases, removed eggs from the female in order to observe their development. The eggs developed normally; therefore, fertilization must have already taken place at this stage.

S te p 4 (Figs $2-3$ to $2-7$, and $3-3$ to $3-7$ ): The male releases the female's chelifores and pushes her backwards with all his ambulatory legs. Then, he brings his ovigerous legs towards those of the female and transfer of the eggs is performed. The female moves her ovigerous legs actively (with 4 distal segments of her ovigerous legs), in order to place the eggs onto the base of the ovigerous legs of the male. During this process, the male turns the female upside-down so that both face each other, heads in opposite directions. After the egg transfer, male and female separate.

$S$ te p 5 (Figs 2-8, 3-8): The male moves its ovigerous legs actively. He secretes mucus from the cement glands in the femur of each ambulatory leg, and covers the eggs with that mucus. Then, he pulls out the mucus-covered eggs one by one from the mucus pool around the base of the ovigerous leg, using 4 distal segments of his ovigerous legs. He forms a braceletlike structure with each egg at the tip of each mucus thread, originating from a mucus ring around the leg (Fig. 5-1). These mucus structures solidify in about $24 \mathrm{~h}$.

We have stained females with mature eggs with Nile blue or neutral red in order to color-mark the eggs (blue or red). The eggs kept the color until they developed into free-swimming larvae (Nakamura, unpublished). The stained females were kept together with unstained males, and it was found that a male could hold red and blue eggs on the same ovigerous leg. However, eggs in the same bracelet were stained in the same color. From this fact, it is clear that the eggs in one bracelet came from one female, but the bracelets on the same ovigerous leg need not originate
1

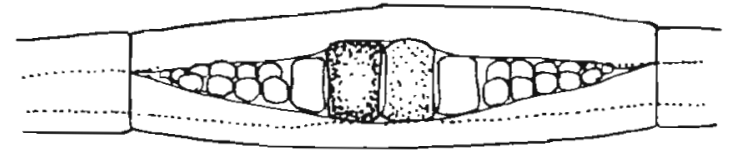

2

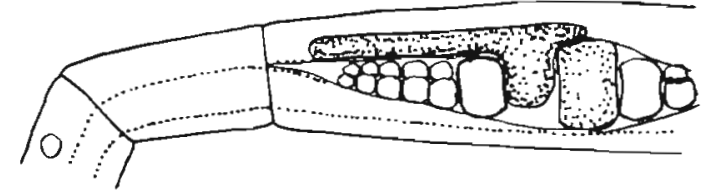

3

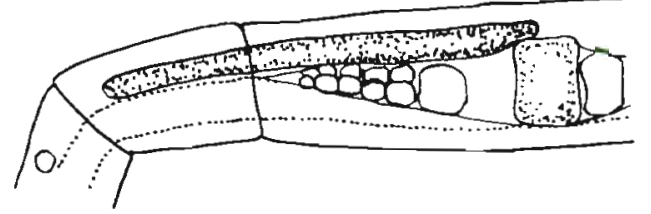

4

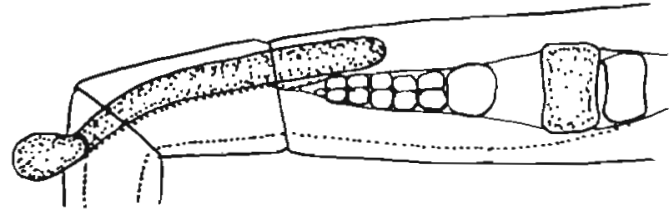

Fig. 4. Propallene longiceps. 1: Female femur with 2 mature eggs (dotted); $2-4$ : release of 1 mature egg
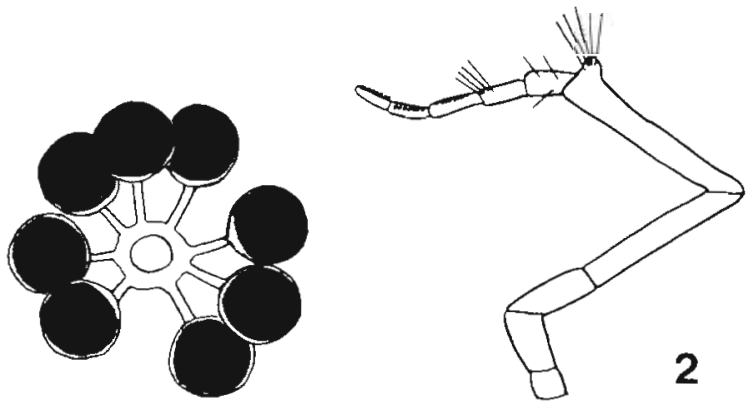

1

Fig. 5. Propallene longiceps. 1: bracelet-like egg mass; 2: ovigerous leg of male. Note a knob-like swelling on the 5th segment

from the same female. In the laboratory, it was rather unusual for one male to mate with only one female exclusively.

Usually, the eggs from left-side legs of one female form one egg mass, the eggs from right-side legs another separate egg mass. When transferred to the male, the eggs are translocated to the ovigerous leg on the identical side of the female leg. Consequently, at the end of copulation, the male holds one egg mass on each ovigerous leg.

The egg is deformed and sometimes flat immediately after the oviposition, but it becomes almost 
spherical within an hour. The egg shell originates from the mucus fluid secreted by the male and has a stalklike thread at one end. The egg attaches at this end to a ring on the ovigerous leg of the male. The egg shell is soft and sticky immediately after it is formed, thus, the eggs stick together if they are removed soon after eggmass formation.

The ovigerous males collected by dredging usually held 4 to 6 of the bracelet-like egg masses on each ovigerous leg; the maximum observed was 11. Each bracelet had usually 8 eggs, although there was a range from 5 to 10 . Among several egg masses on one ovigerous leg, the one in the distal position contains the furthest developed embryos (Nakamura, unpublished). This is due to the habit of the male to place newly transferred eggs on the basal portion of its ovigerous legs. The fifth segment of the ovigerous leg of the male has a knob-like swelling which probably prevents the loss of egg bracelets (Fig. 5-2).

\section{DISCUSSION AND CONCLUSIONS}

Previous reports contained only a few suggestions about copulation and oviposition of pycnogonids (Hoek, 1881; Cole, 1901; Sanchez, 1959). In the present study, we report more than 30 cases of copulation and oviposition observed under laboratory conditions. All individuals studied behaved in the same way. Therefore, the information presented appears to characterize a general pattern of copulation and oviposition of Propallene longiceps.

Oviposition and, probably, fertilization are performed while the male is sitting on the back of the female, assuming a ventral-to-dorsal position. This position seems suitable for the female to collect the eggs laid without interference by her partner. The change in position to ventral-ventral seems necessary for transferring the eggs laid from the female to the male. All eggs laid were transferred. In contrast, in Anoplodactylus lentus some of the eggs laid remain on the female's ovigerous legs (Cole, 1901).

Cole (1901) observed copulation of Anoplodactylus lentus in the morning, while Sanchez (1959) noticed it generally at night. We observed copulation of Propallene longiceps mainly at night, but sometimes during the day. There were no special factors, such as time of day, light or temperature, affecting copulation and oviposition in P. longiceps.

Concerning the duration of copulation, Hoek (1881) stated that it took about half an hour in Phoxichilus laevis. According to Cole (1901) it lasted only about 5 min in Anoplodactylus lentus. In contrast, Jarvis and King (1972) found that copulation took quite a long time, sometimes 5 weeks, in Pycnogonum littorale, but only a few hours in Nymphon gracile. In Propallene longiceps, it required several hours to complete the entire process

According to Sanchez (1959), all eggs matured at the same time in the femur of Endeis spinosa, while only 2 eggs matured in Callipallene species. We found that only 2 eggs matured at any time in a femur of Propallene longiceps. According to Jarvis and King (1972) all eggs mature and are released from a femur at one time. The number of eggs being laid at a given time seems to vary in different species.

Regarding egg-mass formation, King and Jarvis (1970) reported for Nymphon gracile that the male gathers the eggs laid with its ovigerous legs. In Propallene longiceps, the female collects the eggs laid with its ovigerous legs and transfers them to those of the male. The male then forms a bracelet-like egg mass, usually consisting of 8 eggs. When there are many bracelets of egg masses on one leg, the distal ones are in advanced development as compared with those on the proximal leg part. A male can receive eggs from many females at various times and always places the new ones at the base of its ovigerous legs.

When blue or red stained mature females were cultivated together with unstained males, it was found that one male could acquire both blue and red egg masses. This fact documents that the male received eggs from two or more females. Since Propallene longiceps mates and lays eggs under laboratory conditions, this pycnogonid seems to provide good material for studying embryonic development. Observations on early embryonic development are underway.

Acknowledgement. We thank Mr Hajime Ueda of Shimoda Marine Biological Station, The University of Tsukuba, for excellent technical assistance.

\section{LITERATURE CITED}

Cole, L. J. (1901). Notes on the habits of pycnogonids. Biol. Bull. mar. biol. Lab., Woods Hole 2: 195-207

Hoek, P. P. C. (1881). Report on the Pycnogonida dredged by H. M. S. Challenger during the years 1873-76. Rep. scient. Results Voyage HMS Challenger (Zool.) 3: 1-167 
Jarvis, J. H., King, P. E. (1972). Reproduction and development in the pycnogonid Pycnogonum littorale. Mar. Biol. 13: $146-154$

King, P. E., Jarvis, J. H. (1970). Egg development in a littoral pycnogonid Nymphon gracile. Mar Biol. 7: 294-304
Sanchez, S. (1959). Le développement des pycnogonides et leurs affinités avec les arachnides. Archs Zool. exp. gén. 98: 1-101 Trav. Stn biol. Roscoff

Sekiguchi, K., Nakamura, K., Konuma, S. (1971). Egg-carrying habit and embryonic development in a pycnogonid, Propallene longiceps. Zool. Mag. 80: 137-139

This paper was presented by Professor M. Anrakui it was accepted for printing on November 14, 1979. 